\title{
NONCOMMUTATIVE JORDAN ALGEBRAS OF CHARACTERISTIC 0
}

\author{
R. D. SCHAFER ${ }^{1}$
}

Jordan algebras are commutative algebras satisfying the identity

$$
\left(x^{2} a\right) x=x^{2}(a x) .
$$

These algebras have been studied extensively.

A natural generalization to noncommutative algebras is the class of algebras $A$ satisfying (1). Linearization of (1), if the base field contains at least 3 elements, yields

$$
(x y+y x, a, z)+(y z+z y, a, x)+(z x+x z, a, y)=0
$$

where $(x, y, z)$ denotes the associator $(x, y, z)=(x y) z-x(y z)$. If $A$ contains a unity element 1 , and if the characteristic is $\neq 2$, then $z=1$ in (2) implies

$$
(y, a, x)+(x, a, y)=0,
$$

or, equivalently,

$$
(x a) x=x(a x) \text {. }
$$

That is, $A$ is flexible (a weaker condition than commutativity). If a unity element is adjoined to $A$ in the usual fashion, then a necessary and sufficient condition that (2) be satisfied in the extended algebra is that both (2) and (3) be satisfied in $A$.

We define a noncommutative Jordan algebra $A$ over an arbitrary field $F$ to be an algebra satisfying (1) and (4). These algebras include the best-known nonassociative algebras (Jordan, alternative, quasiassociative, and-trivially-Lie algebras). In 1948 they were studied briefly by A. A. Albert in [1, pp. 574-575], ${ }^{2}$ but the assumptions (1) and (4) seemed to him inadequate to yield a satisfactory theory, and he restricted his attention to a less general class of algebras which he called "standard." In this paper, using Albert's method of traceadmissibility ${ }^{3}$ and his results for trace-admissible algebras, we give a

\footnotetext{
Presented to the Society, October 30, 1954; received by the editors August 18, 1954.

1 This research was supported in part by a grant from the National Science Foundation.

2 Numbers in brackets refer to the references cited at the end of the paper.

${ }^{3}$ We use the formulation given in [2] as being more convenient for characteristic 0 than the modified version presented in [4]. We are limited to the characteristic 0
} 
structure theory for noncommutative Jordan algebras of characteristic 0 .

1. Some elementary properties. It is shown in [1] that flexibility implies that (1) is equivalent to any one of the following:

$$
x^{2}(x a)=x\left(x^{2} a\right),(x a) x^{2}=x\left(a x^{2}\right),(a x) x^{2}=\left(a x^{2}\right) x,
$$

and that (1) and (5) imply that $A$ of characteristic $\neq 2$ is Jordanadmissible; that is, the commutative algebra $A^{+}$in which multiplication is defined by $x \cdot y=(x y+y x) / 2$ is a Jordan algebra.

It seems not to have been noted that every flexible Jordan-admissible algebra of characteristic $\neq 2$ satisfies (1), so that $A$ is a noncommutative Jordan algebra if and only if $A$ is flexible and Jordanadmissible. For, denoting right and left multiplications in $A$ by $R_{x}$ and $L_{x}$ respectively, $\left(x^{2} \cdot a\right) \cdot x=x^{2} \cdot(a \cdot x)$ implies

$$
\left[R_{x^{2}}+L_{x^{2}}, R_{x}+L_{x}\right]=0 \text {. }
$$

But (4) implies $\left[L_{x}, R_{x}\right]=0$, while (3) implies both $\left[L_{y}, R_{x}\right]=\left[R_{y}, L_{x}\right]$ and

$$
R_{x y}-R_{x} R_{y}=L_{y x}-L_{x} L_{y}
$$

In particular, we have $\left[L_{x 2}, R_{x}\right]=\left[R_{x 2}, L_{x}\right], R_{x 2}-R_{x}^{2}=L_{x 2}-L_{x}^{2}$. Then (6) gives $0=\left[R_{x 2}+L_{x 2}, R_{x}\right]+\left[R_{x 2}+L_{x 2}, L_{x}\right]=\left[2 L_{x}^{2}-L_{x 2}+R_{x}^{2}, R_{x}\right]$ $+\left[2 R_{x 2}-R_{x}^{2}+L_{x}^{2}, L_{x}\right]=2\left[L_{x 2}, R_{x}\right]+2\left[R_{x 2}, L_{x}\right]=4\left[L_{x 2}, R_{x}\right]$, implying (1). It is not possible to derive (4) from Jordan-admissibility even for algebras containing a unity element, for there are known examples of Jordan-admissible algebras which are not flexible, but which do contain $1[3$, p. 186].

Any noncommutative Jordan algebra of characteristic $\neq 2$ is powerassociative. ${ }^{4}$ For, defining $x^{k+1}=x^{k} x$, we may prove $x^{\lambda} x^{\mu}=x^{\lambda+\mu}$ by induction on $\lambda+\mu=n$. Since this is true for $n=2,3$ by (4), we assume $x^{\lambda} x^{\mu}=x^{\lambda+\mu}$ for all $\lambda+\mu<n, n \geqq 4$. Let $a=x^{n-3}$ in (5): $x x^{n-1}=x^{n-2} x^{2}$ $=x^{n-1} x=x^{n}$. We need to prove $x^{n-\alpha} x^{\alpha}=x^{n}$ for $\alpha=1, \cdots, n-1$, and prove this by proving $x^{n-\alpha} x^{\alpha}=x^{n}=x^{\alpha} x^{n-\alpha}$ by induction on $\alpha$. This has been proved for $\alpha=1$, and we assume $x^{n-\beta} x^{\beta}=x^{n}=x^{\beta} x^{n-\beta}$ for all $\beta \leqq \alpha<n-1$, and prove $x^{n-(\alpha+1)} x^{\alpha+1}=x^{n}=x^{\alpha+1} x^{n-(\alpha+1)}$ as follows. Let

case by the fact that, for an absolutely primitive idempotent $u$ in a general (commutative) Jordan algebra $A$ of characteristic $p>0$, the structure of $A_{u}(1)$-the subalgebra on which $u$ acts as an identity-is not known. When this result is known, it may yield not only a determination of (commutative) Jordan algebras of degree one, but also, by Albert's refinement in [4] of the trace-admissibility technique, a structure theory for noncommutative Jordan algebras of characteristic $p>0$.

1 This is proved for characteristic $\neq 2,3,5$ in $[1$, p. 574]. 
$a=x^{n-(\alpha+2)}, y=x, z=x^{\alpha}$ in (2). Since the sum of any three or fewer exponents is $<n$, we have $0=2\left(x^{2}, a, x^{\alpha}\right)+4\left(x^{\alpha+1}, a, x\right)=2 x^{n-\alpha} x^{\alpha}$ $-2 x^{2} x^{n-2}+4 x^{n-1} x-4 x^{\alpha+1} x^{n-(\alpha+1)}=4 x^{n}-4 x^{\alpha+1} x^{n-(\alpha+1)}$. But (3) implies $0=2\left(x^{\alpha}, a, x^{2}\right)+4\left(x, a, x^{\alpha+1}\right)=4 x^{n}-4 x^{n-(\alpha+1)} x^{\alpha+1}$.

2. Trace-admissibility. A bilinear function $\tau(x, y)$ on a powerassociative algebra $A$ and with values in the base field $F$ is called an admissible trace function for $A$ (and $A$ is called trace-admissible) in case

(i) $\tau(x, y)=\tau(y, x)$,

(ii) $\tau(x y, z)=\tau(x, y z)$,

(iii) $\tau(e, e) \neq 0$ for any idempotent $e$ of $A$,

(iv) $\tau(x, y)=0$ if $x y$ is nilpotent.

If $A$ contains 1 , then the use of a linear function $\delta(x)=\tau(1, x)$ may be substituted for that of $\tau(x, y)$, and it is known [2, p. 319] that (i)-(iv) are equivalent to:

(I) $\delta(x y)=\delta(y x)$,

(II) $\delta((x y) z)=\delta(x(y z))$,

(III) $\delta(e) \neq 0$ for any idempotent $e$ of $A$,

(IV) $\delta(x)=0$ if $x$ is nilpotent.

TheOREM. Any noncommutative Jordan algebra $A$ of characteristic 0 is trace-admissible.

Proof. Since any subalgebra of a trace-admissible algebra is traceadmissible, it is sufficient to prove this theorem under the assumption that $A$ has a unity element 1 . It is well known that, since the characteristic is 0 ,

$$
\delta(x)=\text { Trace } R_{x}^{+}=(1 / 2) \text { Trace }\left(R_{x}+L_{x}\right)
$$

is an admissible trace function for the Jordan algebra $A^{+}$. We shall show that $\delta(x)$ in (8) is also an admissible trace function for $A$.

Since powers in $A$ and $A^{+}$coincide, (III) and (IV) are valid in $A$ because they hold in $A^{+}$. Flexibility implies (I), for $\delta(x y)-\delta(y x)$ $=(1 / 2)$ Trace $\left(R_{x y}+L_{x y}-R_{y x}-L_{y x}\right)=(1 / 2)$ Trace $\left(R_{x} R_{y}-R_{y} R_{x}\right.$ $\left.+L_{y} L_{x}-L_{x} L_{y}\right)=0$ by (7). Now (II) in $A^{+}$implies

$$
\delta((z \cdot y) \cdot x)=\delta(z \cdot(y \cdot x)) \text {. }
$$

Hence (3), (I), and (9) yield $4 \delta((x y) z)=\delta(2(x y) z+2(x y) z)=\delta(2 x(y z)$ $-2(z y) x+2 z(y x)+2(x y) z)=\delta(4 x(y z)-x(y z)-(y z) x-(z y) x-x(z y)$ $+z(y x)+(y x) z+(x y) z+z(x y))=4 \delta(x(y z))-4 \delta((z \cdot y) \cdot x)+4 \delta(z \cdot(y \cdot x))$ $=4 \delta(x(y z))$, implying (II).

3. Structure theory. Because of the general results in [2], the structure theory for noncommutative Jordan algebras of characteristic 0 
may be given as a set of corollaries to our theorem in $\$ 2$. Define the radical $N$ of $A$ to be the maximal nilideal ${ }^{5}$ of $A$, and $A$ to be semisimple if its radical is 0 . Define $A$ to be simple if $A$ is simple in the ordinary sense and not a nilalgebra.

CoROLlaRY 1. The radical $N$ of any noncommutative Jordan algebra $A$ of characteristic 0 is the set of all $z$ satisfying $\delta(x z)=0$ for every $x$ in $A$, and coincides with the radical of the Jordan algebra $A^{+}$. Also $A / N$ is semisimple.

Corollary 2. Any semisimple noncommutative Jordan algebra $A$ of characteristic 0 is uniquely expressible as a direct sum $A=A_{1}$ $\oplus \cdots \oplus A_{t}$ of simple ideals $A_{i}$.

COROLlaRY 3. The simple noncommutative Jordan algebras of characteristic 0 are

(a) the simple (commutative) Jordan algebras,

(b) the simple flexible algebras of degree two,

(c) the simple quasiassociative algebras.

The simple quasiassociative algebras are determined in $[1$, Chap. V] and are further studied in [5]. A set of necessary and sufficient conditions for the multiplication table of any simple flexible algebra of degree two is given in $[1$, p. 588] but, inasmuch as these algebras include such interesting examples as the Cayley-Dickson algebras and the $2^{t}$-dimensional algebras obtained by the Cayley-Dickson process [6], a complete determination of those which are not commutative remains an interesting problem.

\section{REFERENCES}

1. A. A. Albert, Power-associative rings, Trans. Amer. Math. Soc. vol. 64 (1948) pp. 552-593.

2. - A theory of trace-admissible algebras, Proc. Nat. Acad. Sci. U.S.A. vol. 35 (1949) pp. 317-322.

3. - New simple power-associative algebras, Summa Brasiliensis Mathematicae vol. 2 (1951) pp. 183-194.

4. - The structure of right alternative algebras, Ann. of Math. vol. 59 (1954) pp. $408-417$.

5. C. M. Price, Jordan division algebras and the algebras $A(\lambda)$, Trans. Amer. Math. Soc. vol. 70 (1951) pp. 291-300.

6. R. D. Schafer, On the algebras formed by the Cayley-Dickson process, Amer. J. Math. vol. 76 (1954) pp. 435-446.

The University of Connecticut

5 One cannot hope to prove that this radical is nilpotent, or even solvable. For every Lie algebra is its own radical by this definition. 